\title{
PLASTICITY AS A PHYSICAL-CHEMICAL PROCESS OF DEFORMATION OF CRYSTALLINE SOLIDS
}

\author{
Kurapov G. ${ }^{1 \mathrm{a}}$, Lezhnev S. ${ }^{2 b}$, Orlova E. ${ }^{1}$, Volokitina I. ${ }^{1 \mathrm{c}}$, Tolkushkin A. ${ }^{2 \mathrm{~d}}$ \\ ${ }^{1}$ Kazakh national technical university after K.I. Satpaev, Almaty \\ ${ }^{2}$ Karaganda state industrial university, Temirtau \\ akurapov1940@mail.ru, ${ }^{b}$ sergey_legnev@mail.ru, cirinka.vav@mail.ru, ${ }^{\mathrm{d}} \mathrm{mrgugimon@gmail.com}$
}

Keywords: plasticity, crystalline solids, deformation.

\begin{abstract}
Conducted a series of experiments of upsetting of steel samples. Theoretically developed and experimentally verified a fundamentally new way to control the properties of the processed metal by changing the rigidity factor of the system processed material - deforming tool.
\end{abstract}

\section{Introduction}

The nature of plasticity and fracture of solid materials is studied in different branches of science, ranging from geomechanics and ending with nanotechnology. In geomechanics discusses the process of moving from geoplatform with their consequences in the form of mountain building, earthquakes, volcanic activity, to offset the mountain layers with the problems of securing mining. The scale of manifestations of plasticity range from $10^{7} \mathrm{~m}$ to $1 \mathrm{~m}$, and naturally recognizes the physical and chemical processes that lead to changes in the composition and structure of the interacting masses.

In solid mechanics the scale of deformable objects varies from $10^{-1} \mathrm{~m}$ to $10^{-10} \mathrm{~m}$. This dimension overlaps the currently fashionable trend - nanostructured corresponding to $10^{-9} \mathrm{~m}$. To the middle of the last century, the majority of authors examined the plasticity of crystalline materials, which include metals and alloys, from the point of shear sliding mechanism of one of the planar surfaces relative to the others. Developed with half of the last century the theory of dislocations, as moving linear defects in the crystal structures and advanced on this basis, the hypothesis of disclinations as well as the previous hypothesis, has not made a significant refinement in knowledge about the nature of plasticity.

One of the most representative areas of the theory of plasticity - formal physics and mathematics, in which the solution of applied problems of processing materials by pressure is given by the approximation of the laws of elastic deformation in the area of elastic-plastic and large plastic deformations. This approach, in most cases, proved to be quite effective, as allowed, by introducing into the resulting equations of empirical coefficients, solve with necessary for the processing of metals by pressure accuracy for many practical tasks.

In the works of scientific schools of V.A. Likhacheva, V.E. Panina, E.E., Zasimchuka, A.A. Presnyakova was clearly shown that the process of plastic deformation is autowave character, is a generator of dynamic forces and moments in the processing of metals by pressure. Despite considerable research of this schools listed above in relation to real technological processes there were no findings and no recommendations.

Formal understanding of the mechanism of plastic deformation at the same time didn't denied its connection with the change of structure of metals and alloys, one manifestation of which is a texture formed by cold deformation. However, purposefully these issues were examined not as intensive, since it has long been considered that the dominant role in the deformation process play the above mentioned mechanisms, and changes in the structure or appearance of new phases is associated in nature.

Many authors pointed out that the study of the microstructure of deformed materials allows to study only the result and not the process of structure formation. One of the first to the idea that the process of plastic deformation of metals has physic-chemical nature and occurs as a phase 
transformation was expressed by A.A. Bochvarov and Z.A. Swiderskaya [1] during describing discovered by them the phenomenon of superplasticity, and then supported by A. A. Presnyakov, and G.V. Starikova [2]. Visible results were obtained Kravz-Tarnavsky [3], first gained during dynamic deformation of martensitic steel "strip". The most complete analysis of experimental data on structural transformations during the deformation of metals with physic-chemical positions was done in [4-6].

Some researchers have concluded that the phase transition is the main mechanism of plastic deformation, ensuring the possibility of forming a solid body without destruction. Ya.I. Frenkel [7], and then B.N. Malyshev [8] was suggested that the partial presence of metal in the solid body in a state of "fusion". Even further went A.A. Presnyakov, which formed a view on the implementation of plastic deformation through the localization by the mean of mechanism "running neck" [6]. Later in conjunction with the U.K. Duysemaliev [9] they explained the concept of "atomic gas" responsible, in their opinion, for the manifestation of superplasticity of metals. And finally, academician V.E. Panin, which is one of the founders of the mesomechanical theory, also came to the conclusion that plastic deformation of solids can only develop localized as local structural transformation in the areas of stress concentrators of different scales [10, 11].

Research of plastic flow of the metal, carried out by the Kazakhstan school of researchers under the guidance of A.A. Presnyakov [6] with photographing of specimens during tension and simultaneous continuous registration of efforts, gave a presentation on the development of plastic flow in metals and alloys, different from the conventional. It was experimentally shown that the initial stage of plastic flow of samples during tension, which had previously considered as stage of uniform flow, flows very unequal. Deformation periodically localized in narrow zones, which can reach enormous values (up to $30000 \%$ ) with the formation of "micro neck" or "mini neck").

\section{Research methodology}

Experiments on creep during tensile of cylindrical samples, performed by a number of authors on a variety of titanium alloys, aluminium and high-alloy steels showed [12] that for most metallic materials the dependence of yield strength on the degree of deformation, built in a semilogarithmic or logarithmic coordinates, have a linear character.

The mathematical description of the strain rate for such experimental data was proposed by Y. I. Frenkel [7], considering the plastic deformation as the "liquefaction" of the metal with the formation of the centers or nuclei of the new phase:

$$
\dot{\varepsilon}=\operatorname{Bexp}(-\mathrm{Q} / \mathrm{RT}) \text {, }
$$

where B - coefficient,

$\mathrm{Q}$ - activation energy,

$\mathrm{R}$ - Boltzmann constant,

$\mathrm{T}$ - test temperature.

Conducted in the present work, the experimental studies of the deformation of the cylindrical samples at upsetting confirmed the results obtained by A. A. Presnyakov [7] during tensile. Because plastically deformable body is an open thermodynamic system, the deforming tool is both a source of mechanical energy and heat removal surface from a deformable metal.

If consider the idea that the metal becomes "quasi-fluid", even without explaining the mechanism of the transition in such state border slip cones arising, in the opinion of the Gubkin, during upsetting that must be considered as isotherms, separating areas of high (light - side surface) and hindered (ends) plasticity (Fig. 1). The fact that during dynamic loading of steel samples the structure of this boundary is converted to martensite [3] shows the physic-chemical nature of the mechanism of plasticity of metals. Its implementation is provided by the structural-phase transformations, which develop on diffusion-less mechanism due to the high speed of loading, when the diffusion process is difficult. 


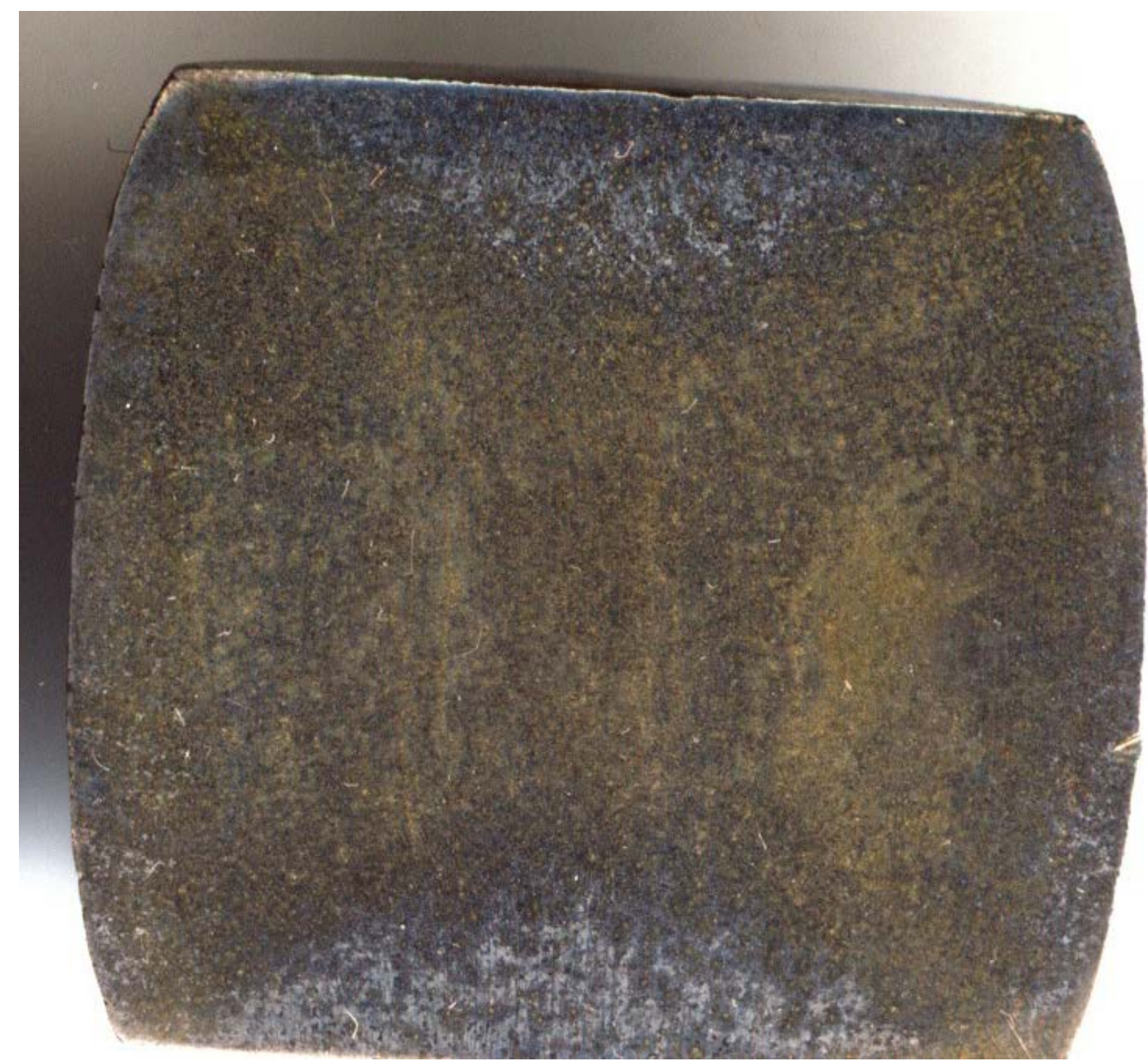

Fig. 1. Closeup of a longitudinal section of upsetted steel sample

\section{Results and discussion}

Below are the results of experiments on upsetting of steel cylindrical samples with a record of external parameters on the camera (Fig. 2). Sample upsetting with the ratio of height to diameter 1:1; 2:1 and 3:1 were carried out on a hydraulic press through a polished plates. The rate of deformation of about $1 \mathrm{~mm} / \mathrm{min}$. Registration of loads conducted using tensoresistive load cells.

As for tension, in the process of upsetting of metal samples in the first stage is the period of "quasiuniform" deformation with forming of "mini neck" and only if the total upset about 30\% begins to develop a common barrel. In addition, in logarithmic coordinates $\lg \varepsilon-\lg \sigma$, where $\varepsilon$ is the deformation rate, and $\sigma$ is the resistance to deformation, dependence of the resistance to deformation from the deformation degenerates into a series of intersecting lines. 


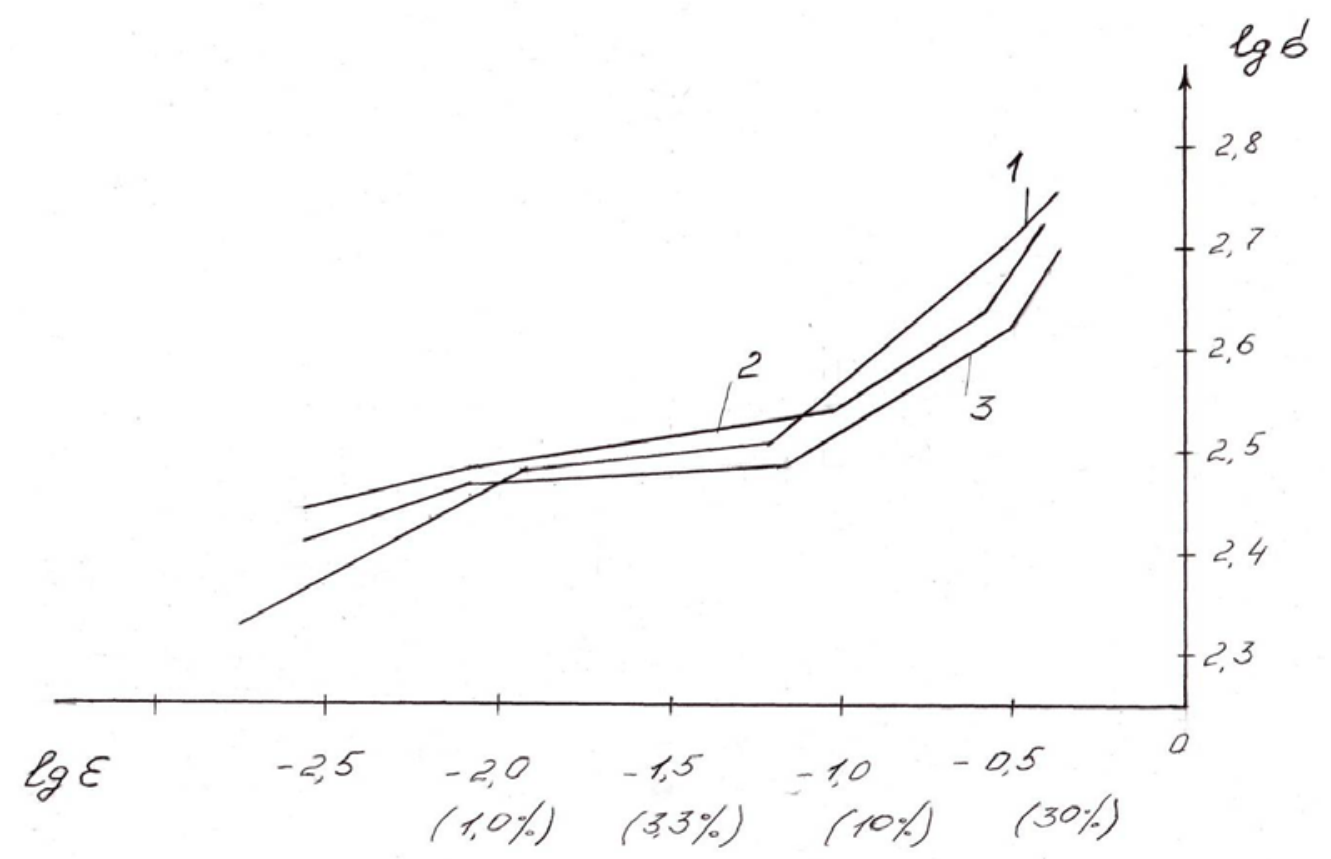

1, 2, 3 - samples with a height to diameter ratio, accordingly 1:1; 2:1 and 3:1

Fig. 2. Dependence of lgo lge.

This behavior depends on the presence of straight line kinks with the accumulating degree of deformation, on the one hand, reflects the special role of certain degrees of deformation in the change of the stress-strain state of the material, and, on the other hand, is a confirmation analogy with linear dependencies obtained in the description of topochemical reactions. As known, topochemical reactions are reactions taking place on a hard surface with the formation of a new phase with the transition from kinetic flow to diffusion, and subsequent to the termination of the reaction. These reactions describe using equation of Avrami-Erofeev-Kolmogorov, having a form similar to the expression (1).

This analogy allows to conclude that the exponential character of the expression (1) is a manifestation of topochemical process of plastic deformation of metals occurring with the formation of a new "quasi-fluid state of metal in the zone of deformation and subsequent termination of this zone. Presumably deformation under tension or compression can be divided into the following areas:

- single acts of plastic deformation, located randomly throughout the length of the sample when "mini necks" or "mini barrels" visually not yet identified ( $\varepsilon$ up to $1 \%$ );

- with forming of «mini necks or mini barrels» ( $\varepsilon$ up to 7\%) - «running necks» according to A.A. Presnyakov;

- with forming of common neck or barrel ( $\varepsilon$ до 30\%);

- destruction ( $\varepsilon$ more than 30\%).

Based on literature data and own experiments, both in laboratory and industrial conditions, proposed the mechanism of plastic flow of metals, combining a number of different approaches to this problem. According to it, the elastic loading of the sample occurs before the moment, where in its cross-section, due to internal elastic distortion of the crystal structure, in the weakest place there is an area with energy sufficient to move from being the original "low-temperature" phase in more "high-temperature". The transition is accomplished by displacement of the atoms of the crystallite in the new position, and this transition is accompanied by a local loss of strength and a kind of "liquefaction - plasticization " of material. The formation of such microscopic (dot) weakening hearth leads to the decrease of the effective cross-section of a nearby solid metal, and thus, to grow in it more stress, contributing ultimately to the distribution of "liquefaction" zone over the entire section of sample. 
Under the influence of external in relation to the zone of liquefaction elastic forces occurs relaxation of loads in elastically deformed zones of the sample and develops viscous deformation in the weakening area of metal. Deformation of this unit area is accompanied by an additional release of energy due to the work of deformation with formation of micro neck.

The process of plastic deformation in the microvolume (microsection) is accompanied by heat removal in the neighboring area. The zone of plastic flow is rapidly cooled, the metal part does reverse transition from $\mathrm{P}_{2}$ to $\mathrm{P}_{1}$, but part of it is fixed in a high-temperature condition of $\mathrm{P}_{2}$. Actually this is a kind of hardening of the material in the area of implementation of plasticity, while the metal is hardened, and the process of plastic flow is terminated.

It is the transition state (quasi-fluid), which is intermediate between the two stable states and responsible for the implementation of the plastic flow of the material without breaking its continuity. In a state of structural-phase transition $\mathrm{P} 1 \rightarrow \mathrm{P} 2$ in the field of plastic flow may be only a part of metal, but sufficient in number and location to ensure proper plastic flow.

According to works of E.E. Zasimchuk [13], the formation of the zone of weakening occurs instantaneous. However, it would be more correct to assume that the propagation velocity of the weakening zone in the cross section of the sample is equal to the speed of propagation of longitudinal elastic waves in the material studied, i.e. the speed of sound in this material. The loss of strength in the zone of plasticity is accompanied by a dynamic reduction of the elastic part of the sample, plastic flow of weakening metal and wave of stress relaxation along the axis of the sample.

However, the wave of relaxation with relation to the primary (longitudinal) acting perpendicularly or at an angle to the axis of the sample, is the transverse which velocity is equal to the product of the material speed of sound multiplied by the Poisson's ratio ( 0.2 to 0.3 for metals). This is the reason that registered by V.E. Panin with employees [14], the speed of the stress relaxation waves several times less than the speed of sound in the test material. Indirect confirmation of this fact is experimental evidence that under the additional imposition on the deformation during tensile of various waves - from sound to high-frequency ranges - the most accurate results are always obtained by superposition of waves across action of tensile forces [15].

Since the "solid" areas of sample (non-deformable), boundary with area of a single strain, are the surfaces of the heat removal, occur heat transfer from the zone of plasticity in these zones, which are in direct contact with them. The reverse transition of metal in the zone of deformation from "quasi-liquid" - plastically deformed state in solid - occurs as a result of structural-phase transformations with corresponding recrystallization of the original structure. Moreover, the crystallization centers are border crystallites of the solid phase.

In work [16] experimentally shown that during upsettling of the metal samples, forming crystallites with size close to the value of the elastic deformation in the direction of the force and with sizes close to the size of the original crystallites in the plane transverse to the action of a deforming force. The increase of the grain surface of the substance and partial quenching of the high-temperature phase leads to hardening of the zone of plastic deformation in comparison with the initial state (deformation-phase hardening).

Actual value of total change of sample size ${ }_{\Delta} \mathrm{l}_{\mathrm{a}}$ in the process of a single act is determined by the elastic deformation of its parts outside the zone of plasticity ${ }_{\Delta} \mathrm{l}_{\mathrm{o}}$, the elastic deformation of the tool and deforming machine ${ }_{\Delta} \mathrm{l}_{\mathrm{m}}$, as well as the amount of movement of the working tool during a single act of deformation ${ }_{\Delta} \mathrm{l}_{\mathrm{t}}$ :

$$
{ }_{\Delta} \mathrm{l}_{\mathrm{a}}={ }_{\Delta} \mathrm{l}_{\mathrm{o}}+{ }_{\Delta} \mathrm{l}_{\mathrm{m}}+{ }_{\Delta} \mathrm{l}_{\mathrm{t}} .
$$

The ratio of the values included in this expression defines a single change of sample size for a single act of deformation.

In lab tests, under conditions where the rigidity of the deforming device and tools much more than rigidity of test sample at low loading rates, the basic quantity that determines the movement of metal in the area of weakening is the elastic deformation of the sample parts out of plasticity area ${ }_{\Delta} \mathrm{l}_{\mathrm{o}}$. This value is determined by the modulus of elasticity of the sample material and the yield strength, and for most metals is approximately equal $l_{0} \cdot 10^{-3}$. 
For most technological machines (presses, rolling mills and so on) components of equation significantly affect on the total displacement in a single cycle of deformation. This is due to the fact that technological machines are designed for large volume of production of metal and they are made to limit the total load (including safety margin). The characteristics included in equation (2) become comparable. But then change "rigidity" parameter of deforming machine and tool will change the size of the zone recrystallization with appropriate changes in the structure, strength and ductility of a deformable metal.

It should be kept in mind that the rigidity, on the one hand, characterizes the amount of elastic load per unit displacement, but on the other hand, - stress relaxation time of load shedding. For low rigidity of machines the speed of the elastic displacement of the parts of the machine lower at the weakening point of the stroke of the deforming tool at the same time will be less, and, accordingly, less will be component ${ }_{\Delta} \mathrm{l}_{\mathrm{m}}$.

The frequency of deformation pulses uniquely associated with the loading rate, with the rigidity of the system "sample - deforming machine" and physic-chemical properties of the sample material. At the moment of the beginning of weakening, the full development of this process and the time until formation of hardened structures are determined by the properties of a deformable material. At the same time, the value of deformation per cycle and the time of load accumulation to the maximum value before a new pulse of weakening determined by the mechanical rigidity of the system and the loading rate.

Since the deformation of the sample by machine goes on continuously, then occurs repeated elastic loading with the formation of the next area of plastic deformation, but with some higher strength characteristics than the previous single act. From the areas of plasticity ("quasiliquid") through the boundary surfaces in the form of heat and waves of relaxation on the rest of the metal is transferred additional energy. But, as a rule, at the initial stage of deformation it is not enough that subsequent area of a single cycle of deformation formed next to the previous area with a minimum strength of cross section, in which the most likely development of plastic deformation are randomly placed along the axis of action of the loading force.

However, gradually weakest areas (sections) as the result of structure-deformation recrystallization strengthened and overall strength properties between the individual sections are aligned so that the additional energy in the form of heat or elastic waves enough to subsequent act of weakening occurred near the previous one. Consistent flow of plastic deformation in a restricted zone leads to the formation of macro-regions of centered form changing with a noticeable reduction of the cross section compared to the original, i.e. to the formation of the common neck during tensile or common barrels during upsetting.

Further deformation process, registered on the indicator curves until reaching the maximum value of the load consists of sequential formation of running necks (during tensile) or barrels (during upsetting) along the length of the sample. And only after a significant hardening of the metal, leading to averaging its mechanical properties establishes conditions when in the process of sequential deformation comes such a huge part of metal that formed a single neck (during tensile) or single barrel (during upsetting), in which occurs the most notable change of section until fracture.

Elementary calculations show that during the deformation of the standard steel samples with a diameter of $10 \mathrm{~mm}$ and a tensile speed of $1 \mathrm{~mm} / \mathrm{min}$, the residence time of the metal in a plastic state is just $0.0013 \%$ of the total time, and the rest of the time samples are in solid state and deforming elastically. Therefore, the reason that the rheological calculation methods of plasticity of metals do not give data close to the experimental is the fact that during defining the viscosity characteristics of the metal used methods, in which metal is mostly in the elastic state.

In the regular processes, which include rolling, drawing and pressing through the die, metal alternately passes through all degrees of deformation. At sufficiently high speeds in the area of the instrument may form a single zone of plasticity.

One of the factors that decisively determine the state of the material in the zone of deformation is rigidity of the system "machine - tool - deformed sample". It is the elastic deformation of this system determines the work done in the area of weakening during the single cycle. The existence of 
metal in a plastic state is determined by the heat removal out of this area, and the amount of heat depends on the work of deformation. Therefore, the lesser deformation in a single cycle, the lesser time metal stay in a plastic state and the lesser secondary grain.

Since displacement in weakening zone carries elastic deformation determined by the rigidity of the system, then, the lesser the rigidity of the system, the lesser elastic displacement during a single act, the work of deformation and the size of the secondary grains of crystallites. Another effect during reducing the rigidity of the system is increasing the intensity of hardening of metal as a result of refinement of the crystallites and the residue of the high-temperature phase. During the experiment, this is manifested as increased deformation loads with decreasing rigidity of the system and the immutability of the other conditions of the experiments.

Outlined above position [16] allows to refuse from notions about the deformation zone as a consequence of the mechanical movements of parts of the crystallites relative to each other, from the theories of lattice defects as carriers of plasticity (theory of dislocations and disclinations) and to recognize the physic-chemical nature of the plastic deformation. This mechanism is inherent to all types of metal forming and cutting. These same patterns are evident during the deformation of nonmetallic crystal structures, i.e. is the same for the plasticity of all solid crystalline systems [17].

\section{Summary}

1. Plastic flow of metals (solid crystalline materials) occurs in very localized areas where the metal is in a non-equilibrium transition state. Localization can occur cyclically in different parts of a deformable material or, under certain conditions, to unite in one common deformation zone.

2. The formation of localized deformation area is accompanied by local heat, deformation waves, sound manifestation, electromagnetic effects, exzoemission of electrons and other particles, up to neutron radiation, which indicates the underlying physic-chemical phenomena in metals during plastic deformation.

3. Deformation mechanism passes through the transition phase with the liquefied metal in the zone of plasticity and as a result we observe a structure obtained by recrystallization. Because during a single pulse occurs direct and inverse phase transitions, some of the material remains in the form of high-temperature phase. The decrease in grain size during recrystallization and the presence in the material of a certain amount of high-temperature phase leads to the strengthening of this area, and in general to the work hardening. The mechanism of plastic deformation is topochemical and described by equation of I. I. Frenkel and Avrami-Erofeev-Kolmogorov.

4. The change in the rigidity factor of the system with any method of deformation changes the nature of the metal flow. This is reflected in the structure of the cross-section of the processed metal, in the location of zones of active and difficult to deformation, the change in the structure of the metal, especially in the zone of active deformation.

5. All factors affecting on deformable metal, contributing to the growth of the work of deformation for a single cycle and increase the temperature in the zone of plasticity contribute to a longer residence time of the metal in the transition (plastic) state and to increase the size of the crystallites observed as a result of plastic flow. All factors contributing to the reduction of the deformation in a single cycle of weakening and lowering the temperature in the zone of weakening will lead to reduction of the sizes of the secondary crystallites.

6. Based on the earlier proposed ideas about the mechanism of plastic deformation of metals theoretically developed and experimentally verified a fundamentally new way to control the properties of the processed metal by changing the rigidity factor of the system processed material deforming tool that allows a wide range to change the grain size of the processed metal, and therefore its properties. The process can be easily implemented in any production, as it requires minimal changes to existing production machines. 


\section{References}

[1] A.A. Bochvar, Z.A. Sviderskaya, The phenomenon of superplasticity in zinc and aluminum alloys, Izvestiya Akademii Nauk SSSR, 1945, 9, p. 821-824.

[2] G.V. Starikova, Investigation of superplasticity in metal alloys, Author's abstract of the master's thesis, 1963.

[3] V.P. Kravz-Tarnavsky, Specific strip in steel, Journal of Russian Metallurgical Society, 1928, 3, $162 \mathrm{p}$.

[4] S.I. Gubkin, Plastic deformation of metals, Metallurgizdat, 1961, v.1, 2, 3.

[5] Matters of plastic deformation of the metal, Proceedings of institute after A.A. Baykov, 1962, 9, $236 \mathrm{p}$.

[6] A.A. Presnyakov, Localization of plastic deformation, Nauka, 1981, 120 p.

[7] Ya.I. Frenkel, Collection of selected works, Akademia nauk SSSR, 1958, v.2, 392 p.

[8] V.P. Malyshev, B.T. Abdrakhmanov, A.M. Nurmagambetova, Fusibility and ductility of metals, Nauchny mir, 2004, 148 p.

[9] A.A. Presnyakov, U.K. Duisemaliev, The concept of superplastic flow of metals and alloys, Publishing house « Signet-Print », 2006, 155 p.

[10] V.E. Panin, V.E. Egorushkin, A.V. Panin, D.D. Moiseenko, Nature of localization of plastic deformation of solids, Journal of Technical Physics, 2007, v. 77, 8, p. 62-69.

[11] V.E. Panin, V.E. Egorushkin, P.V. Makarov, Physical Mesomechanics and computer design of materials, Nauka. Siberian Publishing House of the Russian Academy of Sciences, 1995, v. 1, 298 p.

[12] Sh.Sh. Ibragimov, E.S. Aitkhozhin, S.K. Kusainov, Yu.S. Pyatiletov, E.V. Chumakov, temperature dependence of radiation creep of aluminum, Izvestiya Akademii Nauk KazSSR, Physics and Mathematics series, 4, 1983, p. 55-56.

[13] E.E. Zasimchuk, L.I. Markasheva, Microstrip in nickel monocrystals deformed by rolling, Naukova dumka, 1998, 36 p.

[14] V.E. Panin, Yu.V. Grinyaev, V.E. Egorushkin, The spectrum of excited states and mechanical vortex field in a deformed crystal, Izvestia VUZ. Physics, 1987, 1.

[15] G.P. Metaksa, G.G. Kurapov, A.A. Vinnitsky, A.A. Presnyakov, Copyright certificate №722357. A method of processing metals and alloys, 12.09.1977.

[16] V.K. Kezhaliev, T.V. Chernoglazova, N.N. Mofa, A.S. Degtyareva, G.G. Kurapov, E.N. Suleymenov, Localization of plastic deformation and nonequilibrium structural deformation transformation, Selected Works, Publ. «Complex», 2004, 271 p.

[17] M.V. Chaykina, Mechanochemistry of natural and synthetic apatite, Publ. RAS, 2002, 232 p. 